\title{
Odontólogos: ¿Cuántos somos y en que lugar de Chile ejercemos nuestra profesión?
}

\section{Dentists: How many are in Chile and where we exercise our profession?}

\author{
Karla Moscoso-Matus ${ }^{1 *}$, Iris Espinoza-Santander ${ }^{2}$
}

1. Unidad de Auditoría Interna, Servicio Médico Legal, Santiago, Chile

Facultad de Odontología. Universidad San Sebastián. Sede Puerto Montt

Correo:kmoscoso@gmail.com

2. Directora de Centro de Epidemiologia y Vigilancia de las Enfermedades Orales (CEVEO)

Facultad de Odontología de la Universidad de Chile Correo: iespinoz@u.uchile.cl

${ }^{*}$ Correspondencia a: Dra. Karla Moscoso-Matus Unidad de Auditoría Interna, Servicio Médico Legal, Santiago, Chile

Correo: kmoscoso@gmail.com

Trabajo recibido el 17/03/2017. Aprobado para su publicación el 27/06/2017

\section{Sr Editor}

Las redes sociales se han constituido en un elemento poderoso de acción social, difundiendo y entramando lazos que tal vez en otras circunstancias no existirían. No es extraño que por ellas circule todo tipo de información. Así, por ejemplo, durante el año 2016 en el ámbito odontológico circularon por el ciberespacio variadas noticias relativas a la cantidad de odontólogos presentes en nuestro país:

A mitad de año, encontramos un documento con logo del Colegio de Dentistas AG, pero sin fecha ni firmas, que hace referencia a la necesidad de conformar el capítulo Regional Metropolitano del Colegio. Lo interesante del documento, es que menciona la existencia de 2.835 dentistas colegiados en la Región Metropolitana, de un total de 4.558 colegas colegiados, es decir el $62,1 \%$, constituyéndose en uno de los escasísimos documentos que muestra datos de georeferenciación de la distribución de odontólogos a lo largo del país, pero que sólo incluye a odontólogos colegiados. También encontramos el estudio del Dr. Cousiño(1), del año 2013, quien, vía Servicio de Impuestos Internos, estimó la existencia de 18.115 odontólogos. Sin embargo, este dato es distinto al obtenido vía Superintendencia de Salud por Moscoso(2), quien determinó la existencia de 15.091 odontólogos registrados a septiembre 2014, sólo un año después. Y finalmente, durante el 2015, dada la sobreoferta laboral de odontólogos por el número creciente de universidades que dictan la carrera de odontología, ADEO (Asociación de Estudiantes de Odontología de Chile) publicó en su página de Facebook copia de una carta enviada a la Ministra de Educación solicitando acreditación obligatoria para la carrera de Odontología en todas las universidades donde esta se imparte.

Contribuyendo a la incertidumbre de los datos antes expuestos, número de odontólogos en Chile y su distribución por regiones, así como reales necesidades de seguir aumentando este número, exponemos dos hechos adicionales: En primer lugar, la información contenida en el repositorio de datos "Global Health Observatory" de la Organización Mundial de la Salud, informa en su página web(4) que el número de dentistas en Chile era <0,05 por 10.000 en el año 2009 (indicando como fuente de información al Fondo Nacional de Salud), lo que significaría 16.990 odontólogos en total en Chile según población estimada por INE para el mismo año ${ }^{(5)}$; dato definitivamente erróneo. En segundo lugar, el hecho más reciente, durante la campaña de marketing de la Universidad San Sebastián para invitar a postular a nuevos estudiantes de odontología 2016-2017, se publicó una propaganda que mencionaba una relación odontólogos por población de $89 \times 100.000$ habitantes $^{(3)}$, y que, a la larga, ante las críticas de la comunidad odontológica, les significó retractarse.

¿Cuál es la relevancia de toda la informacion anterior?, que sincera el desconocimiento existente respecto al número y la distribución de los profesionales cirujano-dentistas en Chile. Esta carencia de información, nos impide efectuar una correcta planificación estratégica del recurso humano transformando en algo intuitivo una gestión que debiese ser en extremo racional.

Lo lamentable del asunto es que la herramienta para obtener esta información existe, y es el Registro Nacional de Prestadores Individuales de la Superintendencia de Salud(6) pero, tal como se ha mencionado por otras autores ${ }^{(2)}$, este se encuentra subutilizado y desactualizado. Peor aún, el reglamento que lo mandata ${ }^{(7)}$ expresamente en su artículo $\mathrm{N}^{0} 11$ explicita que el registro contendrá: "región o ciudad en la que habitual y predominantemente ejerce sus funciones", asimismo en su artículo $N^{\circ} 5$ numeral A.1 se explicita que "(...)en el mes de enero de cada año, los prestadores individuales de salud que, durante el año inmediatamente anterior, hubieran mudado de región o ciudad en la que habitual y predominantemente ejercian sus funciones, deberán comunicar a la Intendencia de Prestadores de Salud esa nueva información (...)" así como en el artículo $n^{\circ} 9$ se indica que "habrá un Registro Regional de Prestadores Individuales de Salud por cada una de las regiones en que se encuentra dividido el país (...)".

Desconocemos las razones por las cuales no se ha implementado en plenitud todas las características del registro, así como también a quien se le debe exigir el cumplimiento en pleno de él. Asimismo, somos conscientes que la solicitud de un par de ciudadanas tal vez no sea suficiente para que se implemente esta medida, razón por lo cual ésta debe ser una exigencia de los muchos a quienes esta falta de información los 
afecta directamente. Nos gustaría escu char a todos, los odontólogos y estudiantes de odontología, colegio profesional, organizaciones y redes sociales, exigiendo más y mejor información de cuántos somos y en qué lugar de Chile ejercemos nuestra profesión. Esta información es imprescindible para la elaboración de políticas públicas, el análisis de la empleabilidad, la investigación sobre el rol del acceso a la atención odontológica como determinantes de la salud bucal en Chile, en resumen, para mejorar mejor salud bucal de la población.

Las autoras declaran no poseer fuentes de financiamiento Las autoras declaran no poseer conflicto de intereses.

\section{Bibliografía}

1. Cousiño, S. ¿Cuántos somos actualmente los dentistas en Chile? La burbuja odontológica. Contraángulo. [internet]. 2013. [citado: 07 febrero 2017]. Disponible en: http://www.contraangulo.com/2013/07/cuantos-somos-actualmente-los-dentistas.html 2. Moscoso-Matus K., Smok Vásquez P., Botto Beytía AM. Odontólogos del Registro Nacional de Prestadores Individuales de Salud. Rev. Clin. Periodoncia Implantol. Rehabil. Oral [Internet]. 2016. Abril. [citado: 07 febrero 2017]. 9(1): 42-47. Disponible en: http://www.scielo.cl/scielo.php?script=sci_arttext\&pid=S0719010720160001000 $08 \&$ Ing $=$ es.

3. Radio Bio-Bio. Noticias on-line. [internet]. Santiago. Chile. Radio Bio-Bio. [citado: 07 febrero 2017]. Disponible en: http://www.biobiochile.cl/noticias/nacional/ chile/2017/01/06/colegio-de-dentistas-acusa-a-la-u-san-sebastian-de-publicidadenganosa.shtml.

4. Word Health Organization. [internet]. WHO. Global Health Observatory (GHO) data. Workforce. Density of dentistry personnel. 2016 [citado: 27 octubre 2016]. Disponible http://apps.who.int/gho/data/node.country.country-CHL
5. Instituto Nacional de Estadísticas. Gobierno de Chile. [internet]. Santiago, Chile. Estadísticas Vitales. Informe Anual 2009. [citado 3 de noviembre 2016] Disponible en http://www.ine.cl/canales/chile_estadistico/demografia_y_vitales/estadisticas vitales/2011/Vitales_2009_21102011.pdf

6. Superintendencia de Sälud. Gobierno de Chile. [internet]. Registro Nacional de Prestadores Individuales. Santiago, Chile. [citado 20 de noviembre 2016] Disponible en: http://www.supersalud.gob.cl/servicios/576/w3-article-5587.html

7. Ministerio de Salud. Gobierno de Chile. [internet]. Decreto $N^{\circ} 16$. Reglamento sobre los registros relativos a los prestadores individuales de salud, 2009. [citado 21 de noviembre 2016]. Disponible en: www.supersalud.gob.cl/normativa/571/ articles-5057_recurso_1.pdf 